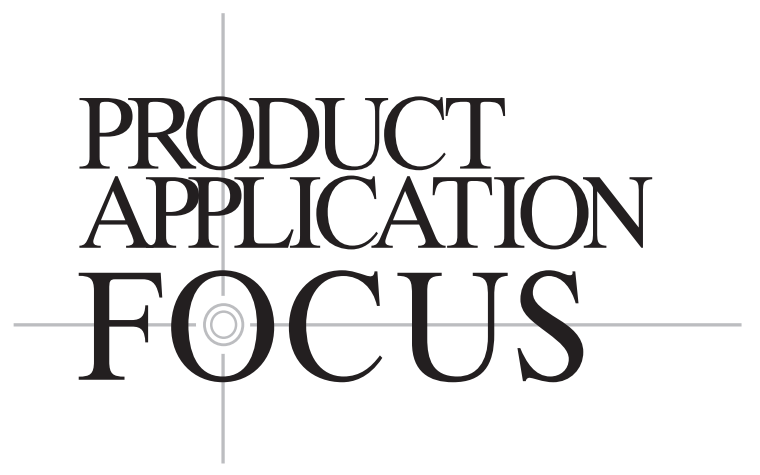

\title{
In-Process Monitoring of Protein Purification with Thin Film Silicon Sensor Technology
}

\author{
Anna Ettinger, Rachel Ostroff, Jeanne Brien, and Barry Polisky \\ Thermo BioStar, Boulder, CO, USA
}

BioTechniques 32:934-938 (April 2002)

\begin{abstract}
We have developed a rapid and sensitive thin film assay for inprocess monitoring of target protein purification. This novel biosensor method provides rapid (5-min) visual evaluation of column purification fractions. The method can be used to monitor the efficiency of purification and potential loss of protein if the column binding capacity is exceeded. The eluted fractions containing the highest yield of target protein can be quickly identified, pooled, and processed.

This convenient platform, known as the SILAS ${ }^{T M}$ product, is a thin-film detection technology in which specific molecular interactions are transduced into visible color changes based on changes in the optical thickness of layers on a silicon surface. The results are interpreted without instrumentation. Proteins eluted from a purification column are adsorbed to the assay surface, and the ligand of interest (target) can be identified with specific binding reagents. Here we demonstrate two protein purification applications for the SILAS technology product: monitoring antibody elution from a Protein $G$ column and evaluating the efficiency of purification of a glutathione$S$-transferase (GST)-tagged recombinant protein through each step of the purification process.
\end{abstract}

\section{INTRODUCTION}

Rapid, simple, and specific detection of one or more target proteins during purification or processing steps is a key requirement in many laboratories. Currently, laboratories are forced to use time-consuming analytical tools such as gel electrophoresis to identify the presence of a desired target within specific fractions from the purification step. This paper describes a new application of a unique thin film technology that detects specifically bound targets on an optically coated silicon surface. This new technology allows simple, non-in- strumented detection of a given target in 5 min using little of sample in the analysis. Thus, this technology addresses a key need for many laboratories. The SILAS ${ }^{\mathrm{TM}}$ product is available from Thermo BioStar (Boulder, CO, USA; product information available at www.silas.thermobiostar.com).

In this technology, the optical thickness of the surface increases when molecules are specifically bound, resulting in a visible color change that is due to destructive interference-specific reflected wavelengths in the light (6). Thin film formation is enhanced when the bound target is recognized by a receptor conjugated to HRP, followed by a precipitation event that results in mass deposition on the surface. The increased optical thickness of the surface results in a visible color change from gold to purple (Figure 1). The optical surfaces and materials are designed to respond to small thickness changes $(10 \AA)$.

This technology has been applied to rapid point-of-care testing for the detection of infectious disease pathogens. Recent clinical studies comparing thin film assay detection of group A streptococcus or influenza virus to culture have demonstrated assay sensitivity to be equivalent to or more sensitive than routine bacterial or viral culture $(1,3)$. Limits of detection as low as $12 \mathrm{pM}$ of a specific analyte have been reported (8).

Non-instrumented, qualitative results are easily determined visually with this thin-film technology. Quantitative results can be obtained by measuring either color contrast with a CCD camera and image processing software (6) or thin film thickness by ellipsometry $(9,10)$.

The thin film technology is a flexible detection platform that has been applied to the detection of multiple analyte types including protein, nucleic acid, and carbohydrate targets (2-4). The smooth silicon surface, with less than a $15 \AA$ surface roughness, accommodates many sample types that are incompatible with the more convoluted membrane assay surfaces. Samples such as viscous respiratory secretions, particu- 
late fecal specimens, and whole blood have been used without observing nonspecific binding $(1,2,8)$. The lack of nonspecific binding to these surfaces allows specimens to be tested without sample filtration or centrifugation and provides a high signal-to-noise ratio.

Here we describe new applications for thin film technology and the new SILAS product for monitoring protein purification in-process. SILAS surfaces are optically coated silicon surfaces coated with an attachment polymer to facilitate protein adsorption. These surfaces can be used to rapidly evaluate protein purification efficiency and track the elution of specific proteins from chromatography separations. The assay format is simple and results in rapid detection of molecules of interest.

\section{MATERIALS AND METHODS}

\section{Materials}

T-polymer (polydimethylsiloxane) was purchased from United Chemical Technologies (Bristol, PA, USA). Poly (PheLys) and Anti-rabbit IgG conjugated to HRP were purchased from Sigma (St. Louis, MO, USA). Anti-glutathione S-transferase (Anti-GST) antibody, HiTrap ${ }^{\circledR}$ Protein G (1 mL), PreScission $^{\mathrm{TM}}$ Protease, Glutathione Sepharose ${ }^{\mathrm{TM}} 4 \mathrm{~B}$, pGex plasmid (pGex 6P-1) vector were purchased from Amersham Biosciences (Piscataway, NJ, USA). The $2 \times Y$ T medium was from BD Biosciences (Palo Alto, CA, USA). Rabbit anti-goat IgG, HRP conjugated, was from KPL (Gaithersburg, MA, USA). Rabbit sera for IgG purification was obtained from Strategic Biosolutions (Ramona, CA, USA). Tetramethylbenzidine (TMB) was from BioFX Laboratories (Randallstown, MD, USA). IPTG and complete ${ }^{\mathrm{TM}}$ Protease Inhibitor Tablets were from Roche Applied Science (Mannheim, Germany). Novex $^{\circledR} 4 \%-10 \%$ Tris-Glycine Precast gels $(1.0 \mathrm{~mm} \times 15$ wells) and Coomassie ${ }^{\circledR}$ SimplyBlue ${ }^{\mathrm{TM}}$ SafeStain were from Invitrogen (Carlsbad, CA, USA). HRP conjugates were prepared by the sodium-metaperiodate conjugation procedure, as previously described (2,7). All reagents were analytical-grade purity.

\section{Antibody Purification}

The IgG purification was performed using a HiTrap Protein $\mathrm{G}$ column. The column was equilibrated with PBS, $\mathrm{pH}$ 7.0, then $2.5 \mathrm{~mL} 10$ times diluted serum were applied to the column. The column was washed with $1 \times$ PBS and total IgG was eluted with $0.1 \mathrm{M}$ Glycine buffer, $\mathrm{pH} 2.7$. The $\mathrm{pH}$ of all fractions was neutralized with $1 \mathrm{M}$ Tris buffer, $\mathrm{pH}$ 9.0.

\section{Recombinant Fusion Protein Purification}

The fusion protein being purified is a ribosomal protein of Neisseria gonorrhoeae linked to GST. The expression vector utilized in the production of this fusion protein is a pGex plasmid (pGex 6P-1) vector containing the ribosomal protein gene (pGex 6P-1 + GC). An overnight culture of E. coli BL21 transformed with pGex $6 \mathrm{P}-1+\mathrm{GC}$ was grown in $2 \times \mathrm{YT}$ media with ampicillin. The culture was expanded, and overexpression of the GC-GST fusion protein was induced with IPTG at a concentration of $100 \mathrm{mM}$ for $4 \mathrm{~h}$. After induction, the cells were pelleted followed by sonication. The sonicated lysate containing inhibitor cocktail was applied to a Glutathione Sepharose 4B column (10 mL) and washed with $1 \times$ PBS until the $\mathrm{A}_{280}$ of fractions returns to baseline. GC-GST fusion protein was eluted using $10 \mathrm{mM}$ glutathione in $50 \mathrm{mM}$ Tris- $\mathrm{HCl}$ buffer, $\mathrm{pH} 8.0$, and appropriate fractions were pooled. This pooled fraction was dialyzed against $50 \mathrm{mM}$ Tris, $\mathrm{pH} 7.0$, containing $150 \mathrm{mM} \mathrm{NaCl}, 1 \mathrm{mM}$ EDTA, and $1 \mathrm{mM}$ DTT, and cleaved with PreScission Protease to release the GST fusion partner from the Neisseria protein. The cleavage product was applied to the same Glutathione Sepharose 4B column. The GC portion of fusion protein was eluted with $1 \times \mathrm{PBS}$, and the GST portion of the protein fusion was removed from the column with the glutathione elution buffer.

\section{Thin Film Surface Construction}

The base substrate of the test surface is crystalline silicon. This base provides a highly reflective, inert, and optically flat surface. Silicon nitride is applied by vapor deposition to a thickness of $515 \AA$. This layer serves as an anti-reflective optical layer. The thickness and refractive index of this layer are controlled so that the surface is gold in appearance. An aminoalkyl T-polymer (polydimethylsiloxane) is applied by spin coating to a final thickness of $135 \AA$. This creates a hydrophobic surface for protein adsorption. Next, the wafers are coated with a layer of Poly (Phe-Lys) $(50 \mu \mathrm{L} / \mathrm{mL} 1 \times$ PBS $)(5,6)$. 


\section{Proteomic Technologies}

\section{Thin Film Assay}

Small aliquots of fractions collected from column chromatography were diluted 1:5 with Protein Diluent $(1 \times$ PBS, $1 \%$ BSA, $0.05 \%$ Tween $^{\circledR} 20, \mathrm{pH} 7.0$ ), and a $30-\mu \mathrm{L}$ volume was incubated on the prepared silicon surface for $1 \mathrm{~min}$ and then washed with PBS, pH 7.4. Proteins from each fraction were thus adsorbed to the surface. A target or protein-specific antibody-HRP conjugate was diluted to $25 \mathrm{ng} / \mathrm{mL}$ with conjugate diluent (0.05 M MOPSO, 5\% BSA, $0.05 \%$ Tween 20, $\mathrm{pH}$ 7.0), and a $30-\mu \mathrm{L}$ volume was incubated on the surface for 2 min, followed by washing with $1 \times$ PBS. Then, $30 \mu \mathrm{L}$ TMB substrate were applied to the surface for $2 \mathrm{~min}$ and washed. All incubations were performed at ambient temperature.

Alternatively, specific protein can be detected after surface adsorption of an unlabeled specific antibody, followed by an anti-species antibody-HRP conjugate and TMB as described earlier.

\section{SDS-PAGE}

SDS-PAGE was performed using 4\%-20\% Tris-Glycine precast gels. Gels were loaded with 3-5 $\mu \mathrm{g}$ protein/lane, run for $2 \mathrm{~h}$ at $125 \mathrm{~V}$, and stained with Coomassie SimplyBlue SafeStain.

\section{RESULTS AND DISCUSSION}

\section{Monitoring Antibody Purification}

The rapid, avid, and high-capacity protein binding properties of the SILAS surface were utilized in an assay designed to monitor serum antibody purification. This 5-min assay provides rapid in-process evaluation of column purification fractions in two steps: binding of the protein mixture to the SILAS surface for $1 \mathrm{~min}$ (Figure 2A) and detection of the pro-

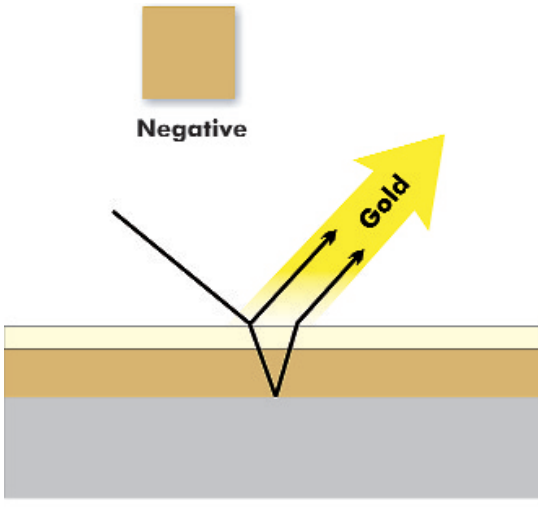

Unreacted Surface

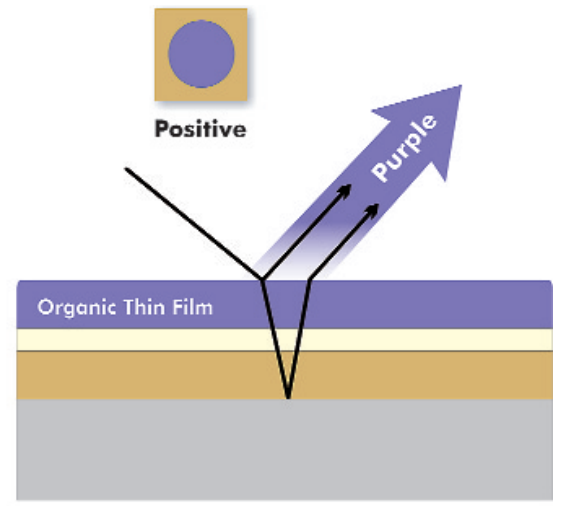

Reacted Surface

Figure 1. Thin film signal generation. Binding of analyte, followed by specific antibody-HRP conjugate and a precipitation reaction on the surface, adds mass to the surface. The additional organic thin film layer causes a color change from gold to purple.
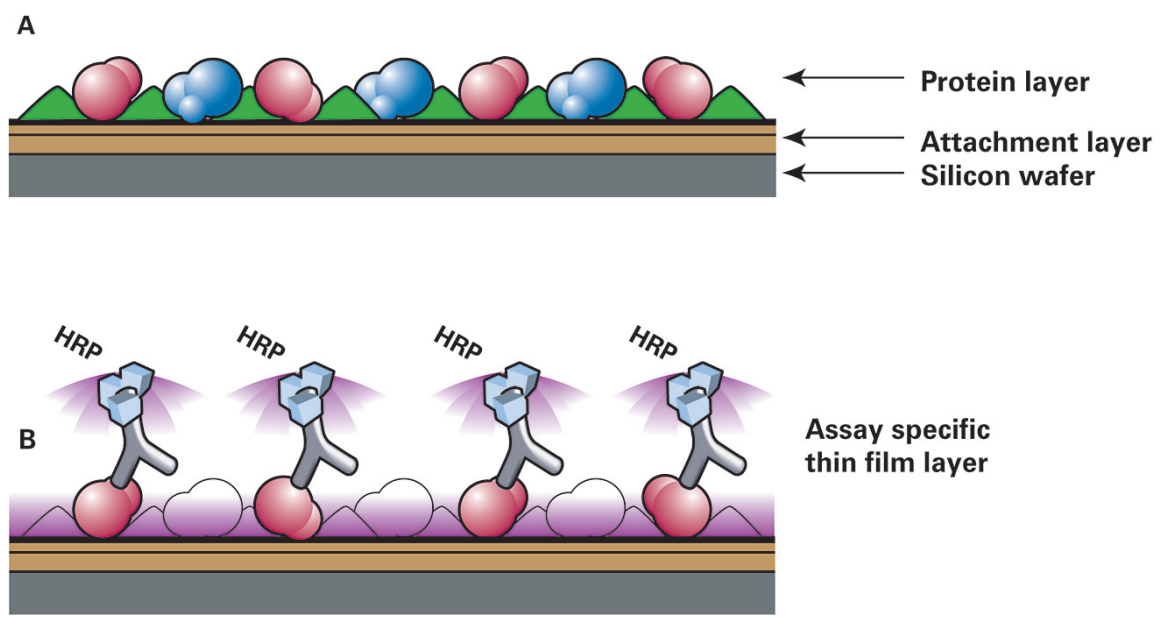

Figure 2. Diagram of the SILAS assay for purification monitoring. (A) Adsorption of protein from the column fraction. (B) Target protein detection and thin film formation enhancement. 
tein of interest following thin film enhancement with a specific antibody-HRP conjugate plus substrate in a 2.2-min reaction (Figure 2B).

The assay can be used to monitor efficiency of purification and the potential for loss of protein if the column binding capacity is exceeded. The eluted fractions containing the highest yield of antibody can be quickly identified, pooled, and processed. Identification of a target protein in the initial column volumes can help to prevent loss of the precious target.

Purification of total IgG from rabbit serum was performed as described earlier, and column fractions were analyzed with the SILAS protein binding surfaces. IgG-containing fractions were identified by the thin film assay using a goat anti-rabbit HRP conjugate and a precipitating HRP substrate. Each column fraction was also analyzed by SDS-PAGE for comparison (Figure 3).

\section{Purification of GST-GC Fusion Protein}

Another application for SILAS technology is monitoring of recombinant fusion protein purification. If specific detection reagents are available for both the fusion tag and the recombinant protein of interest, then purification, proteolytic cleavage, and separation of the two protein components can be monitored. Thus, the efficiency of cleavage and recovery from affinity columns can be quickly evaluated.
The purification of a $N$. gonorrheae (GC) protein fused to a GST tag is described here. Antibody to both the GST and GC components of the fusion protein were available, so two SILAS assays were performed on each column fraction. After induction of the fusion protein expression, the cell lysate was loaded onto a glutathione affinity column to purify the recombinant protein. The recombinant protein is expected to appear in the fractions eluted with free glutathione and not in the flow-through.

The fusion protein was present in both the flow-through and the eluted fractions, as analyzed by the SILAS assay (Figure 4A). These results suggest that the binding capacity of the column was exceeded and recombinant protein leaked through. Obtaining these data while purification is in progress allows for protocol adjustment, re-purification of the flowthrough, and identification of eluted fractions to pool for proteolytic cleavage.

After protease cleavage to remove the GST portion of the protein, the eluted protein was again applied to the glutathione column. If protease cleavage is complete and the GST tagged is removed, then the GC protein would be expected in the flow-through fraction and the cleaved GST portion should be retained on the column. The flow-through and eluted fractions were analyzed with the SILAS assay (Figure 4B). The flow-through fractions analyzed with the GC-specific antibody contained GC protein, as expected. However,

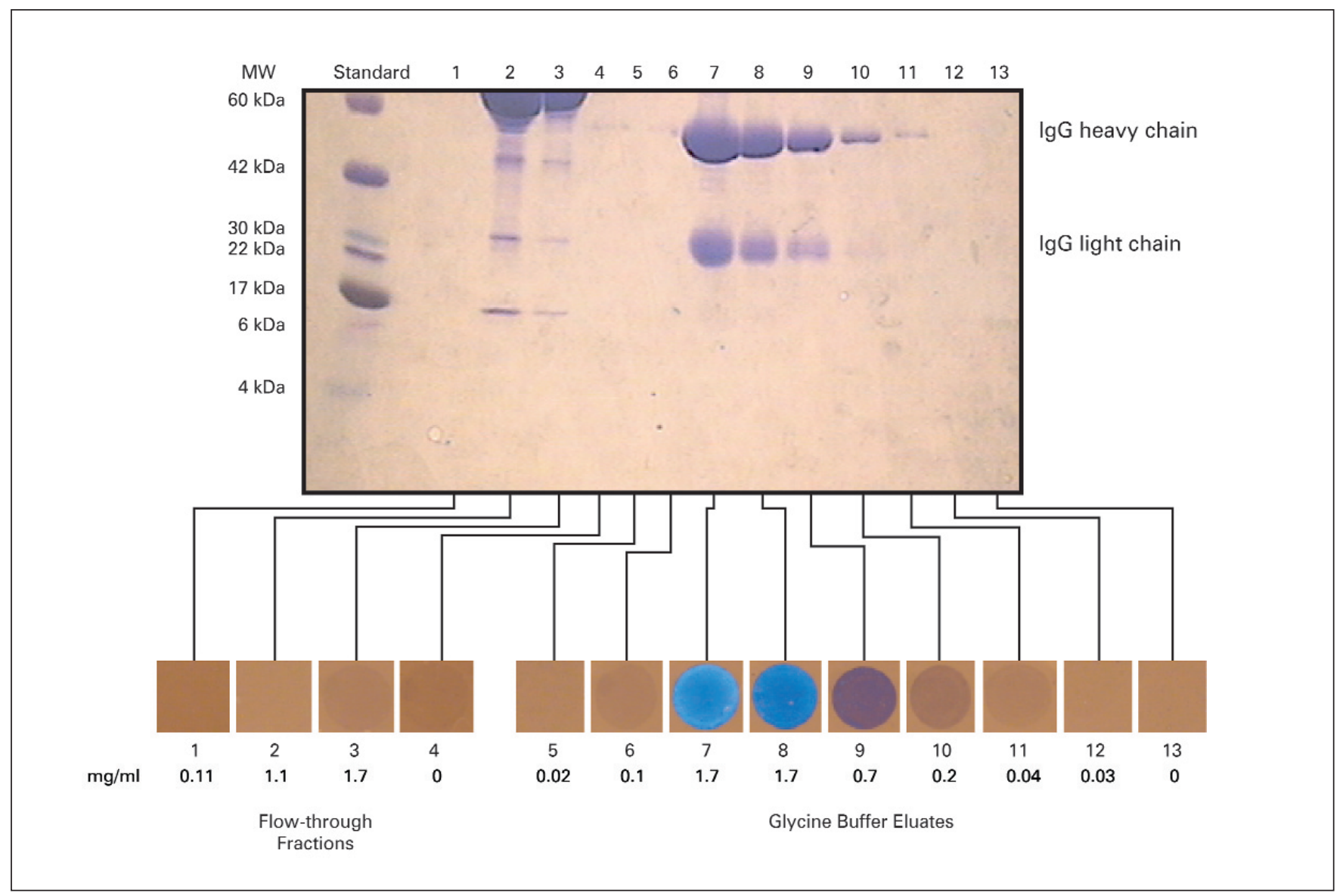

Figure 3. Monitoring antibody purification. Fractions loaded onto SDS-polyacrylamide gels were also analyzed with the SILAS assay, where immobilized antibody was detected. Protein concentration of each fraction is given beneath each chip. 


\section{Proteomic Technologies}
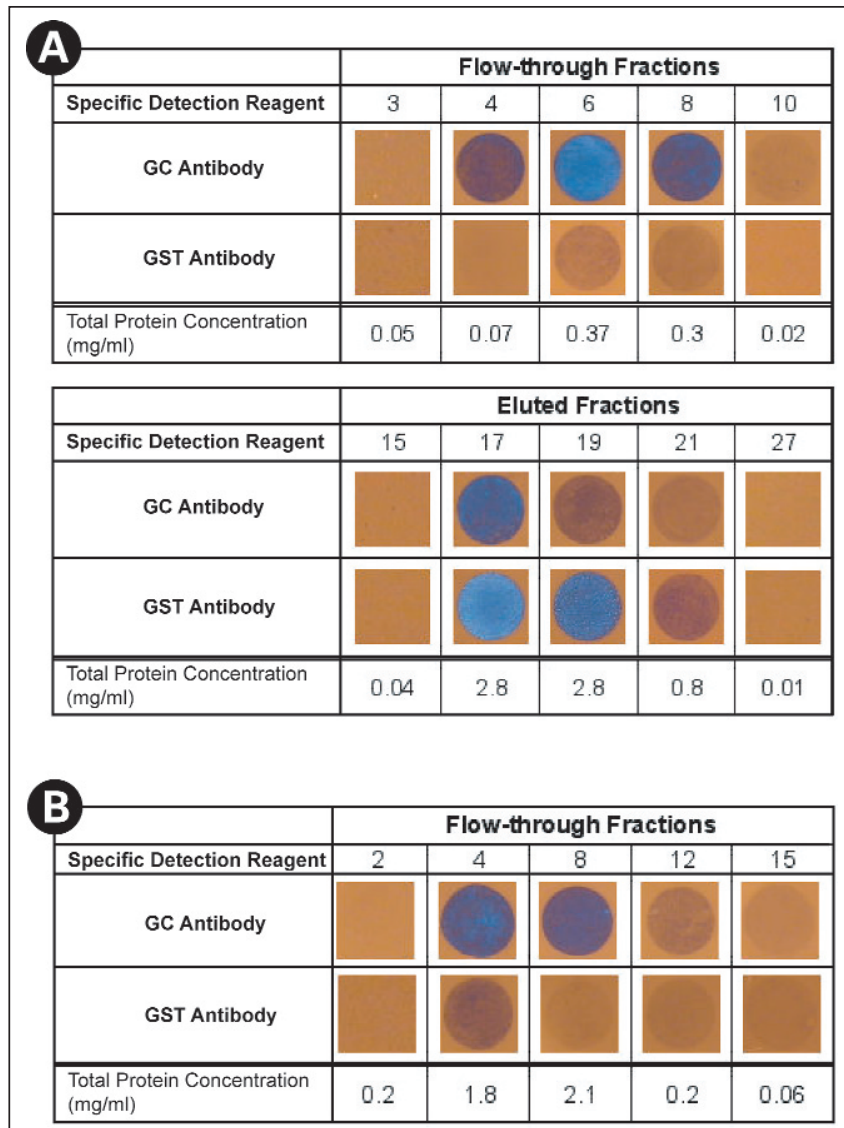

\begin{tabular}{|c|c|c|c|c|c|}
\hline & \multicolumn{5}{|c|}{ Eluted Fractions } \\
\hline Specific Detection Reagent & 17 & 20 & 24 & 26 & 28 \\
\hline GC Antibody & & & & & \\
\hline GST Antibody & & & & & \\
\hline \hline $\begin{array}{l}\text { Total Protein Concentration } \\
(\mathrm{mg} / \mathrm{ml})\end{array}$ & 0.05 & 0.6 & 0.3 & 0.2 & 0.002 \\
\hline
\end{tabular}

Figure 4. Fusion protein purification. Each fraction was analyzed with GCor GST-specific antibody. (A) Fractions from the cell lysate applied to the glutathione column and eluted with free glutathione. Fusion protein is expected in the eluted fractions. (B) Post-protease cleavage analysis of fractions collected from the glutathione column. GC protein is expected in the flowthrough, GST-cleaved portion in the eluate.

some of these fractions also contained significant amounts of the GST fusion, indicating that protease cleavage was not complete and/or the column binding capacity was exceeded. The eluted fractions, expected to only contain GST, also reacted with the GC-specific antibody, confirming that $100 \%$ protease cleavage was not achieved.

The SILAS assay results correlate with gel analysis. Fractions enriched for IgG were easily detected with the SILAS assay in $5 \mathrm{~min}$. Fractions containing other serum proteins do not react with specific antibody in conjugate, and the SILAS results were negative. The SILAS assay quickly identified relevant chromatography fractions for further analysis or processing. All column fractions can be evaluated as they are collected or when fractionation is complete. As only a very small aliquot of each fraction is used in the SILAS assay, the in- process analysis does not significantly impact the final yield of expensive or rare materials.

The rapid SILAS assay can be a useful addition to any protein purification procedure if specific recognition reagents, such as antibodies, are available. Analysis of purification products is rapid and simple, with sensitivity equivalent to SDSPAGE. The thin film assay allows for in-process purification monitoring and selection of appropriate fractions for further experimentation. Loss of protein during purification can be traced, and the SILAS results can be used to evaluate protocol improvements to maximize recovery of the purified product.

The SILAS surfaces provide a rapid method for the detection of analytes of interest. The assays are simple, and no instrumentation is required to interpret the results. Research applications from immunoassay detection to nucleic acid hybridization are easily formatted on this platform and provide a convenient analytical tool.

\section{ACKNOWLEDGMENTS}

We would like to thank Mr. Rob Jenison and Ms. Ayla Haeberli for the help in coating T-poly wafers with PPL. We also wish to thank Drs. John Dorson, Diana Maul, and Gregory Svanas for the critical reading of the manuscript. We are grateful to Mr. Chris High for preparation of the figures.

\section{REFERENCES}

1.Covalciuc, K.A., K.H. Webb, and C.A. Carlson. 1999. Comparison of four clinical specimen types for detection of influenza A and B viruses by optical immunoassay (FLU OIA Test) and cell culture methods. J. Clin. Microbiol. 37:3971-3974.

2.Ettinger, A., A. Laumark, R. Ostroff, J. Brundell, W. Baumgartner, and A. Razumovsky. 1999. A new optical immunoassay for detection of S-100B protein in whole blood. Ann. Thorac. Surg. 68:2196-2201.

3.Gerber, M.A., R.R. Tanz, W. Kabat, E. Dennis, G.L. Bell, E.L. Kaplan, and S. Shulman. 1997. Optical immunoassay test for group A $\beta$ hemolytic streptococcal pharyngitis. JAMA 277:899-903.

4.Jenison, R., A.B. Haeberli, S. Yang, B. Polisky, and R. Ostroff. 2000. Thin film biosensor for rapid detection of mecA from methicillin-resistant Staphylococcus aureus. Clin. Chem. 46:1501-1504.

5.Jenison, R., H. La, A.B. Haeberli, R. Ostroff, and B. Polisky. 2001. Silicon-based biosensors for rapid detection of protein or nucleic acid targets. Clin. Chem. 47:1894-1900.

6.Jenison, R., S. Yang, A.B. Haeberli, and B. Polisky. 2001. Interferencebased detection of nucleic acid targets on optically coated silicon. Nat. Biotechnol. 19:62-65.

7.Nakane, P.K. 1975. Recent progress in the peroxidase-labelled antibody method. Ann. NY Acad. Sci. 254:203-211.

8.Ostroff, R., A. Ettinger, H. La, M. Rihanek, L. Zalman, J.W. Meador, A.K. Patick, S.T. Worland, and B. Polisky. 2001. Rapid multiserotype detection of human rhinoviruses on optically coated silicon surfaces. J. Clin. Microbiol. 21:105-117.

9.Ostroff, R.M., D. Maul, G.R. Bogart, S. Yang, J. Christian, D. Hopkins, D. Clark, B. Trotter, and G. Moddel. 1998. Fixed polarizer ellipsometry for simple and sensitive detection of thin films generated by specific molecular interactions: applications in immunoassays and DNA sequence detection. Clin. Chem. 44:2031-2035.

10.Trotter, B., G. Moddel, R.M. Ostroff, and G.R. Bogart. 1999. Fixedpolarizer ellipsometry: a simple technique to measure the thickness of very thin films. Opt. Eng. 38:902-907.

Address correspondence to Dr. Anna Ettinger, ThermoBioStar Inc., 6655 Lookout Rd., Boulder, CO 80301, USA. email: a_ettinger@thermobiostar.com 